\title{
ESTÍMULOS Y OBSTÁCULOS AL AVANCE DE LA PROPIEDAD CAMPESINA (EL BAIX EMPORDÀ, 1860-1940) ${ }^{1}$
}

ENRIC SAGUER I HOM

Universitat de Girona

\section{RESUMEN}

En este artículo se pretende reflexionar sobre el avance de la propiedad campesina, detectable desde fines del siglo XIX, a partir de una investigación realizada en la comarca catalana del Baix Empordà. Dado que los ejes básicos del proceso fueron la fragmentación y venta de viejos patrimonios rentistas, así como su adquisición - a migajas - por parte de sectores campesinos, se apuntan algunos elementos explicativos tanto del comportamiento de los propietarios rentistas como de los mecanismos que hicieron posible el avance de un grupo significativo de patrimonios campesinos.

\section{ABSTRACT}

The aim of this article is to think about the expansion in peasant property detectable from the end of nineteeth to the middle of twenteeth centuries. The research is centred around one particular Catalan region, the Baix Empordà. The main parts of the process were the break-up and the sale of old estates, as well as its purchase - fragmented - by peasant sectors. Some explanatory elements are set, both related to the behaviour of landed sectors, and to the

${ }^{1}$ Este trabajo forma parte del proyecto de investigación «Factores determinantes del desarrollo agrario en Cataluña: recursos naturales, organización del trabajo y cambio técnico», financiado por la DGICYT (PB96-1157-C03-01). Debo agradecer los comentarios y sugerencias de Ramon Garrabou, James Simpson y los evaluadores de la Revista de Historia Económica, así como de Maurice Aymard, Salvador Calatayud, Rosa Congost, Domingo Gallego y Pere Pascual, miembros del tribunal que juzgó la tesis que ha dado pie a esta publicación. Obviamente los errores del texto sólo son imputables al autor. 
means that make possible the expansion of a significant group of peasant properties.

Investigaciones recientes - y no tan recientes - sobre la evolución de las estructuras agrarias en la Europa Occidental han revelado la inadecuación de la hipótesis kautskiana sobre el triunfo de la gran explotación capitalista ${ }^{2}$ para explicar los procesos de transformación experimentados desde la segunda mitad del siglo XIX hasta el presente. Aunque el mantenimiento de precios agrícolas altos y salarios bajos durante buena parte de la primera mitad de siglo (excluyendo la depresión posnapoleónica) proporcionó una ventaja relativa a las grandes explotaciones, posteriormente ésta fue diluyéndose ${ }^{3}$. A ello contribuyeron la expansión a escala mundial del área de cultivo, la difusión de nuevos medios de transporte y los crecientes efectos de la industrialización sobre el mercado de trabajo. Cada vez existen menos dudas sobre el hecho que el proceso de cambio en las estructuras agrarias desde las últimas décadas del ochocientos no sólo se saldó claramente a favor de las explotaciones de tipo familiar (pequeñas y medianas), sino que, además, se vio reforzado por un significativo flujo de transferencia de propiedades desde sectores rentistas hacia sectores propiamente campesinos. En Inglaterra, este proceso de transferencia llegó a extremos espectaculares durante el intervalo entre la Primera y la Segunda Guerra Mundial, cuando la gran propiedad aristocrática, lentamente acrecentada durante varios siglos, sufrió un brusco retroceso ${ }^{4}$. Procesos de avance de la propiedad campesina, cada cual con sus singularidades, también han sido descritos para Italia ${ }^{5}$, Francia ${ }^{6}$ o Grecia ${ }^{7}$, en algunas ocasiones cargando la pluma con tintes de epopeya. Asimismo, y con los matices que se quiera, distintos estudios regionales españoles han apuntado en la misma dirección ${ }^{8}$, aunque también se hayan constatado procesos de signo contrario en Castilla ${ }^{9}$. Por lo que se refiere a Cataluña, a pesar de que algún autor continúe manteniendo que la

${ }^{2}$ Kautsky (1899).

${ }^{3}$ Koning (1996), pp. 11.39.

4 Thompson (1963), p. 512; Grigg (1989), pp. 98-107, y Goodman y Redclift (1991), pp. 59-65.

5 Massullo (1990), pp. 12-34.

6 Gervais, Jollivet y Tavernier (1976), pp. 193-203.

${ }^{7}$ Karaouzou (1993).

${ }^{8}$ Cela (1979), Calatayud (1989), González de Molina y Sevilla Guzmán (1991), Garrabou (1992) y Grupo de Historia Agraria de Murcia (1992).

'Robledo (1981). 
absorción de la agricultura por el capitalismo dio lugar a una mayor concentración de la tierra ${ }^{10}$, las investigaciones más recientes ${ }^{11}$ confirman la precursora intuición de Yvette Barbaza sobre la Costa Brava ${ }^{12}$; a saber, que si algún cambio se produjo en la distribución de la propiedad de la tierra, éste fue en beneficio de la pequeña y mediana propiedad.

La pretensión del presente trabajo — producto de la tesis doctoral del autor- es reflexionar sobre el proceso de avance de la propiedad campesina a partir de una comarca catalana concreta, el Baix Empordà ${ }^{13}$, y a lo largo del período comprendido entre 1860 y 1940, aproximadamente. Aunque debamos empezar con alguna breve referencia a la magnitud y características de dicho proceso en la zona estudiada, nuestro objeto no será tanto la descripción cuantitativa - tratada en otra parte ${ }^{14}$ - como la exploración de algunas hipótesis explicativas del cómo y del por qué se produjo. Asumiremos como dato y como punto de partida que las transformaciones en la distribución de la propiedad rústica fueron producto,

10 Segura (1993), p. 38.

"Ferrer (1991), pp. 44-49; Tribó (1992), pp. 212-224, y Colomé (1992), pp. 250-254.

12 Barbaza (1988), vol. II, pp. 77-79.

${ }^{13}$ El Baix Empordà es una comarca administrativa situada al NE de Cataluña y con un superficie de $700 \mathrm{~km}^{2}$. El relieve de la comarca combina un llano central de origen aluvial atravesado por dos ríos (Ter y Daró), con una orografía más o menos abrupta en la periferia y un litoral puntualmente escarpado que justifica el apelativo de Costa Brava. Se trata de una zona de clima mediterráneo con una pluviosidad media de $650 \mathrm{~mm}$. anuales. A mediados del siglo xxx la superficie cultivada comprendía, aproximadamente, la mitad del territorio. El resto lo ocupaban los espacios forestales (tuvo particular importancia la zona corchera del macizo de Les Gavarres, que dio lugar a una floreciente industria taponera) y, en menor medida, los pastos. El cultivo predominante era el cereal de secano (70 por $100)$ y, básicamente, los cereales panificables. A la viña y el olivar les correspondía la superficie agrícola restante ( 21 y 8 por 100 , respectivamente). El nivel de especialización era bajo y el destino de la producción el mercado local, cuando no el autoconsumo. Si en algo destacaba el Baix Empordà era en su relativamente elevada densidad ganadera $\left(7.000 \mathrm{~kg} / \mathrm{km}^{2}\right.$, frente a los $4.200 \mathrm{~kg} / \mathrm{km}^{2}$ del conjunto catalán), mayoritariamente caballar y vacuno de labor. La evolución posterior de la estructura productiva acentuó esta especialización pecuaria y forzó la creciente sustitución de los cultivos tradicionales por piensos y plantas herbáceas destinadas al consumo animal. Por lo que se refiere al tamaño de las explotaciones, cabe decir, en primer lugar, que la unidad emblemática era el mas, cuya extensión podía variar entre las 8-10 y las 100-200 hectáreas, en función de su dotación de recursos. En el llano - la zona agrícola por excelencia- pocos masos superaban las 20 ó 30 hectáreas. El mas era también la unidad patrimonial de los grandes patrimonios (integrados por un número variable de masos, generalmente dispersos). En el polo opuesto de la estructura agraria encontramos una constelación de pequeños patrimonios campesinos, también fragmentados y dispersos. Su peso era importante sobre la superficie con uso agrícola (a mediados del siglo XIX el 45 por 100 del suelo agrícola, aproximadamente, era poseído por pequeños propietarios).

14 Saguer (1997). 
básicamente, de las compras y ventas en el mercado de la tierra. Ello nos permite excluir del análisis elementos como las prácticas hereditarias, al menos en un primer momento, y nos fuerza a focalizar la explicación en la doble perspectiva de los oferentes y de los demandantes de tierra. Además, un análisis previo de la dirección de las transferencias mercantiles entre sectores sociales ${ }^{15}$ nos autoriza a focalizar nuestra atención en los dos grupos protagonistas del proceso, a saber: campesinos y hacendados (bisendats) como vendedores; sólo campesinos como compradores. Finalmente sólo cabe advertir que la exploración que vamos a realizar no contempla elementos que, si bien pudieron tener alguna incidencia en el proceso de transferencia de tierra desde sectores rentistas hacia sectores campesinos, no consideramos prioritarios. Así pues, cuestiones como la evolución del precio de la tierra ${ }^{16}$, los efectos del cambio técnico o las estrategias campesinas de acumulación van a quedarse en el tintero.

\section{TRANSFORMACIONES EN LA PROPIEDAD AGRARIA BAJOAMPURDANESA (1860-1940)}

Para el conjunto de Cataluña puede afirmarse que, entre mediados del siglo XIX y mediados del $\mathrm{Xx}$, se dieron claras tendencias hacia la consolidación de la propiedad campesina ${ }^{17}$ y hacia una progresiva

15 Saguer (1996), pp. 494-498.

${ }^{16}$ El análisis de la evolución del precio de la tierra es un terreno todavía poco explorado, en gran medida por las dificultades que presenta la construcción de series de un bien tan heterogéneo como son los derechos de propiedad sobre la tierra. Algunas excepciones notables son las de Robledo (1983), Román (1994) y Sabio (1996). El reciente trabajo de A. Sabio (1996) sobre Cinco Villas (Zaragoza) incide en esta cuestión de la heterogeneidad y pone de relieve una gran dispersión de precios para unidades de tierra con el mismo uso (cereal de secano); dispersión que se atribuye no sólo a diferencias de calidad, sino especialmente a las posiciones de los contratantes en el mercado. Ello permite sospechar que no existe una correspondencia nítida entre la rentabilidad de la tierra como inversión y el precio que ésta registra en el mercado. Por otra parte, la dispersión y el sesgo social que hay tras ella limita el alcance que pueda tener la utilización de precios medios para analizar la actuación de grupos sociales concretos. La inexistencia de series publicadas sobre la comarca analizada o, más en general, sobre alguna zona próxima, las dificultades para realizar personalmente el trabajo y los límites señalados a su utilidad justifican sobradamente que no hayamos dado mayor atención a esta cuestión.

${ }_{17}$ Cabe advertir que el concepto de propiedad campesina está referido a un colectivo - los campesinos - definido de forma relativamente vaga y amplia. En este trabajo entendemos que campesino (pagès) es aquel que trabaja la tierra, aunque no sea ésta su única ocupación. En buena medida este sentido concuerda con uno de los usos que los mismos contemporáneos le daban. $\mathrm{O}$, mejor, con uno de los significados que a partir de 
identificación entre propiedad y explotación, aunque siempre -es importante subrayarlo- dentro de un marco de relativa estabilidad estructural, sin cambios espectaculares. La concreción de dichas tendencias adquirió formas complejas y singulares, de difícil esquematización, en función de un conjunto múltiple de factores: desde el tipo de suelo, la orientación productiva de cada zona y la evolución de los precios, hasta la presión demográfica y la existencia de actividades alternativas o complementarias a la propiamente agraria. Cabe tenerlo en cuenta, pues algunas de las transformaciones que hemos observado en el Baix Empordà no pueden ser elevadas a categoría general para el conjunto catalán. Y lo mismo sucede con las conclusiones extraídas por otros autores en otras comarcas. Un aspecto tan relevante como la variación en el número de propietarios, por ejemplo, registró comportamientos marcadamente distintos. En el Baix Empordà el avance de la propiedad campesina se produjo al tiempo que una significativa reducción del número total de propietarios (del orden del 20 por 100) entre mediados del siglo XIX y el primer tercio del siglo $\mathrm{xx}$, mientras que los estudios realizados para comarcas tan dispares como el Maresme, las Garrigues, el Baix Llobregat, el Penedès o el Bages muestran no sólo un comportamiento de signo contrario, sino incluso -en algunos casos- un espectacular aumento ${ }^{18}$.

Sintetizando un proceso que aquí no podemos describir con detalle, las principales líneas de transformación de la propiedad agraria bajoampurdanesa pueden resumirse como sigue: En primer lugar, como acabamos de indicar, se registró una reducción del número global de propietarios de suelo agrícola, especialmente en los municipios rurales de la comarca. Esta reducción estuvo sin duda relacionada con los procesos de éxodo rural, aunque su vinculación fue más compleja de lo que cabría pensar a primera vista. Paralelamente se experimentó un incremento de la proporción de vecinos propietarios en la mayor parte de localidades

un determinado momento le dieron. A lo largo de la segunda mitad del siglo xx se produjo un cambio significativo de vocabulario en lo referido a las autocalificaciones sociales. A mediados del siglo XIX de pagesos había bien pocos. Sólo aquellos propietarios medianos y arrendatarios (masovers) que disponían de tierra suficiente para dedicarse exclusivamente a la agricultura. Los demás se consideraban a sí mismos como menestrales. Algunas décadas más tarde el significado del término pagès había cambiado. Quienes años antes se consideraban menestrales se habían convertido en pagesos ( $\mathrm{y}$ más tarde en agricultores), sin que las condiciones de su vida material hubieran variado sustancialmente. También en el vocabulario hubo un proceso de campesinización.

18 Llobet (1955), pp. 259-268; Sales (1994), pp. 92-93; Tribó (1992), pp. 216-221; Ferrer (1991), pp. 45-46, y Colomé (1992), p. 252. 
rurales. Aunque a mediados del siglo XIX el grado de acceso a la propiedad de tierra ya era relativamente alto (entre el 70 y 80 por 100 de las unidades familiares poseían tierras en su localidad de residencia), aumentó significativamente en el transcurso de las décadas posteriores. El número de habitantes por cada propietario vecino (el cociente habitantes/propietarios vecinos) pasó de 5,3 a 4,6 entre mediados del siglo XIX y mediados del xx. También retrocedió lo que podríamos calificar de micropropiedad agrícola -aquellos patrimonios que no alcanzaban una hectárea de extensión- especialmente en las zonas que no conocieron avances significativos en la superficie irrigada ni en su densidad urbana. $Y$, por otra parte, a diferencia de lo que sostiene Antoni Segura (1993) en un estudio sobre los propietarios de la tierra en la provincia de Barcelona (cuyas conclusiones eleva a escala catalana), en el Baix Empordà apenas se observa penetración del capital urbano en suelo agrícola. Si bien es cierto que aumentó notablemente el número de propietarios urbanos y la superficie agrícola poseída por este grupo, ello no se debió tanto a compras e inversiones realizadas por sectores burgueses, como a la emigración de grandes $\mathrm{y}$, también, pequeños propietarios hacia villas $\mathrm{y}$ ciudades. El análisis del mercado de la tierra nos ha permitido constatar que no hubo ningún movimiento masivo de inversiones que justifique hablar de penetración de capital urbano ${ }^{19}$.

Este conjunto de procesos adquiere mayor coherencia cuando añadimos que se dieron en un contexto marcado por un cierto retroceso de los grandes patrimonios rentistas. Ésta es, a nuestro parecer, una de las transformaciones más significativas, especialmente en términos cualitativos. Aunque no debamos olvidar que una parte nada menospreciable de hisendats mantuvo, con pocas variaciones, la integridad de sus patrimonios territoriales; tampoco podemos ocultar ni minusvalorar que otra porción no menos relevante vio cómo aquéllos disminuían sensiblemente. De los 33 bisendats que a mediados del XIX tenían tierras en cuatro municipios bajoampurdaneses que hemos estudiado con minuciosidad ${ }^{20}, 17$ (el 50 por 100) llegaron a liquidar la totalidad de sus bienes en dichos municipios. En contraste, los patrimonios rentistas que experimentaron aumentos superficiales fueron comparativamente pocos (ocho en la muestra indicada) y de escasa entidad. Sólo muy parcialmente las pérdidas registradas por los viejos grandes patrimonios repercutieron a favor de otros hisendats de

\footnotetext{
${ }^{19}$ Saguer (1996), pp. 448-454.

${ }^{20}$ Se trata de los municipios de Colomers, Monells, Sant Sadurní de l'Heura y Ullastret.
} 
vieja estirpe. Y la irrupción de nuevos grandes propietarios que, por compra, adquiriesen las tierras de aquellos que liquidaban sus bienes, fue un fenómeno bastante marginal por lo que al suelo con uso agrícola se refiere. En resumen, pues, si existieron movimientos de recomposición en el seno de la propiedad rentista éstos tuvieron un alcance bastante limitado.

La disminución patrimonial de las familias rentistas no debe atribuirse, en términos generales, a procesos de división hereditaria; ni tampoco a la cesión de dominio que implicaban los establecimientos y subestablecimientos enfitéuticos. De hecho, la práctica de las cesiones enfitéuticas, que podría ser considerada una continuación de las estrategias rentistas del setecientos, experimentó una brusca desaceleración a partir de las tres últimas décadas del siglo, en estrecha relación con la paralización del proceso de ampliación del área de cultivo. Sólo permaneció -con sus debidas excepciones- como fórmula asociada al crecimiento de núcleos urbanos. Además, al abandono de la enfiteusis como estrategia rentista se le sumó la progresiva depreciación de los censos monetizados, especialmente a partir de los años de la Primera Guerra Mundial, y un tenue proceso de liquidación de estas cargas a través de su alienación mercantil o su redención por acuerdo mutuo, lo que globalmente contribuyó a una cierta simplificación de la cadena rentista.

La desintegración de los grandes patrimonios se produjo básicamente a través de la alienación de parcelas de tierra mediante su venta en el mercado de la tierra. Los propietarios rentistas vendieron su tierra y lo hicieron, mayoritariamente, a migajas. Hubo pocas ventas de masos completos. Lo habitual fue el goteo de parcelas segregadas de ellos. Ello dio lugar a una progresiva fragmentación de estas unidades de explotación hasta llegar, en bastantes casos, a su desintegración. Ferrer ha constatado algo similar en la Cataluña central ${ }^{21}$. Esta fragmentación podría atribuirse a una estrategia de los propietarios para conseguir un precio superior 0 para retardar la pérdida definitiva de toda una explotación; pero pocas dudas caben que también respondió a las condiciones de mercado imperantes: quienes estuvieron dispuestos a comprar tierra fueron, mayoritariamente, pequeños y medianos campesinos, cuyas posibilidades de acumulación e inversión eran, por otra parte, limitadas. Más adelante plantearemos las condiciones que lo propiciaron. Por el momento, lo que debe quedar claro es que los principales beneficiarios (compradores) de las tierras vendidas por hisendats y grandes propietarios fueron grupos

\footnotetext{
${ }^{21}$ Ferrer (1991), pp. 46-47.
} 
campesinos. En el municipio de Ullastret, por ejemplo, adquirieron el 63 por 100 del suelo vendido por aquéllos, mientras que los grupos propiamente rentistas sólo adquirieron el 31 por 100 de las tierras de sus congéneres. El análisis de los flujos de transferencia de tierras entre grupos sociales confirma a los campesinos como el grupo más activo en el mercado fundiario y, además, como el único que, sin ambivalencias, obtuvo un saldo positivo.

Finalmente sólo debemos advertir que la simplificación de considerar todos los sectores campesinos como un único grupo en el mercado de la tierra no debe ocultar la existencia de situaciones y movimientos divergentes en su seno. No debemos ni podemos olvidar que el avance de algunos patrimonios campesinos también estuvo acompañado por el éxodo y la desposesión de otros. El análisis de la trayectoria patrimonial seguida por las 102 unidades familiares del municipio de Monells que, en algún momento entre 1865 y 1943 , fueron propietarias de tierra en la misma localidad muestra cómo el 45 por 100 redujo su patrimonio territorial, la mayor parte hasta liquidarlo totalmente al tiempo que desaparecían como vecinas de la localidad. Ello también benefició en alguna medida a las familias que permanecieron en el medio rural, la mayor parte de las cuales experimentó sensibles aumentos territoriales.

\section{LA CRONOLOGÍA DEL PROCESO}

Dadas las cambiantes condiciones del sector agrario a lo largo del período considerado, el primer paso que cabe dar para una correcta interpretación, en términos históricos, del proceso de transferencia de tierras a los campesinos consiste en realizar una observación más afinada de sus ritmos y cronología. Aunque ello, por sí solo, no pueda explicar este proceso, nos ayudará a centrar las cuestiones relevantes y nos aportará algunas pistas.

Los datos seriales disponibles indican que, en comparación con períodos posteriores, la movilidad mercantil de la tierra tuvo una intensidad relativamente baja hasta las décadas finales del siglo XIX. Es obvio que los procesos desamortizadores, al menos en sus fases más dinámicas, debieron contribuir a una cierta aceleración de las transferencias de tierra, aunque es oportuno recordar que en Cataluña la superficie rústica desamortizada no fue muy elevada, excepto en puntos muy concretos. En términos generales, tanto para el Baix Empordà como para el conjunto 


\section{GRÁFICO 1}

Cronologia de la venta de tierras de grandes propietarios y de las compras realizadas por campesinos, 1865-1940 (medias móviles de 5 años)

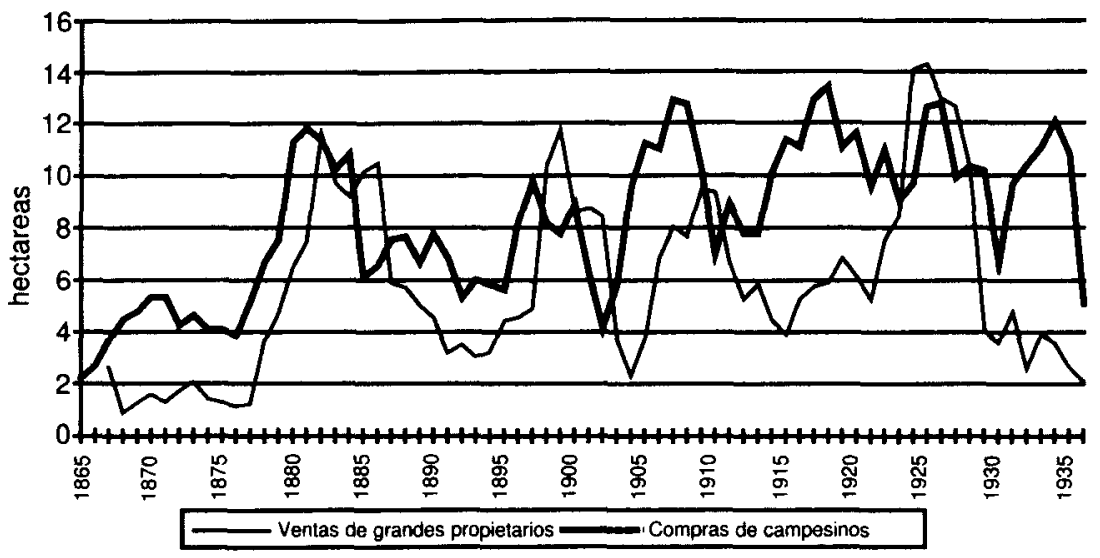

Fuente: Elaborado a partir del Registro de la Propiedad de Monells y Ullastret. La explicación detallada y argumentada de los criterios utilizados para identificar tanto a grandes propietarios como a campesinos puede encontrarse en Saguer (1997). En el trabajo citado también se cuantifican y precisan las magnitudes de un proceso que aquí damos por supuesto.

catalán puede hablarse de una cierta atonía, de una etapa de baja movilidad mercantil, a pesar de existir zonas, especialmente las vitícolas, con un mercado de la tierra más dinámico. Pero incluso en ellas, antes de 1880 , los procesos de venta a cargo de grandes propietarios apenas tuvieron relevancia. Al contrario, en otras zonas se ha demostrado que fueron años propicios para el refuerzo y la expansión de la gran propiedad ${ }^{22}$.

A partir de los años ochenta se abrió una nueva fase durante la cual tendieron a acelerarse las transferencias mercantiles de tierra. Sin embargo, el impacto inicial de la crisis finisecular sobre el mercado fundiario no fue tan unívoco como podría deducirse. Allí donde la filoxera (cuya expansión se dilató a lo largo de las dos décadas de fin de siglo) no destruyó capital fijo, la caída de los precios no tuvo traducción inmediata sobre la distribución de la propiedad. Diversos estudios han verificado que la nota dominante durante la década de los ochenta continuó siendo una

${ }^{22}$ Calatayud (1989) y Millán y Calatayud (1992), pp. 328-334. 
cierta atonía e incluso una ralentización del mercado de la tierra ${ }^{23}$. Pero en el Baix Empordà, si bien el impacto no fue inmediato, sí que parece constatarse una cierta aceleración entre 1880 y 1910, aunque con ciertas ambigüedades y ritmos dispares. Dos hechos destacan particularmente: En primer lugar, no parece que la coyuntura depresiva de aquellos años afectase especialmente a los patrimonios campesinos. Las familias campesinas mostraron una gran capacidad de resistencia y la crisis no se tradujo en un aumento de su oferta de tierras. Por el contrario, y en segundo lugar, se disparó el proceso de venta de tierras procedentes de algunos grandes patrimonios comarcales $y$, paralelamente, las compras realizadas por familias campesinas. ¿Cómo explicar, en un contexto depresivo, la apuesta de muchas familias de labradores y menestrales por acrecentar su patrimonio? Más tarde deberemos aventurar una explicación.

El período realmente clave en el proceso de transferencia de tierras correspondió a los años que rodean la Primera Guerra Mundial ${ }^{24}$. Como advertimos en los párrafos introductorios, ésta no fue una singularidad catalana ni española, sino propia del conjunto europeo. El acceso de los grupos campesinos a la propiedad se intensificó paralelamente al alza de precios de los productos agrícolas en unos términos que los contemporáneos recordaban vivamente incluso bastantes años más tarde. $\mathrm{El}$ volumen de transferencias y el número de operaciones de compraventa aumentaron de forma sensible y generalizada. La presión de la demanda campesina tuvo respuesta entre los grandes propietarios, quienes mostraron una notable disposición a vender, parceladamente, sus tierras. Esta intensidad se mantuvo durante cierto tiempo, aunque en la segunda mitad de la década de 1920 empezaron a percibirse los primeros signos de desaceleración. Ya en los críticos treinta, la tónica dominante se caracterizó por la progresiva pérdida de dinamismo del mercado fundiario $y$, sobre todo, por la pérdida de impulso del proceso de transferencia de tierra a los agricultores. El fracaso en el proyecto de reforma agraria se tradujo en un frenazo de aquello que pretendía impulsar.

Este breve esquema cronológico nos permite situar con mayor precisión el proceso de fragmentación y venta de grandes patrimonios: fue a partir

${ }^{23}$ Calatayud (1989), p. 96, y Tribó (1992), p. 225.

24 Aunque en el gráfico el volumen de tierras transferido decaiga precisamente durante los años de precios más altos, los años circundantes fueron los que registraron mayor número de operaciones y mayor extensión vendida. Por otra parte, la insistencia de algunos testimonios locales en señalar el período de la Primera Guerra Mundial como el momento álgido en el proceso de transferencia mercantil de tierras hacia los campesinos matiza (al alza) la lectura que se obtiene del gráfico. 
de la crisis agraria finisecular cuando estalló. La historiografía ha considerado la crisis agraria finisecular como algo más que una simple coyuntura depresiva. Fue un momento de ruptura y un período clave para el desarrollo del capitalismo en el mundo rural. Aunque el arancel de 1891 frenase la caída tendencial de los precios agrícolas, ésta sólo fue una solución temporal, puesto que se mantuvieron buena parte de los factores que la habían impulsado (mundialización del mercado de productos agrícolas, aparición de productos sustitutivos, aumento de la capacidad productiva de la agricultura europea...). Nada fue igual que antes con posterioridad a la crisis. $\mathrm{Y}$ a sus efectos se sumaron las consecuencias de la creciente subordinación del sector agrario al desarrollo industrial y urbano catalán. La crisis agraria abrió un período de reordenación del sector y de desarticulación de la sociedad rural tradicional ${ }^{25}$. Fue en este contexto cuando la gran propiedad comenzó a fragmentarse y liquidarse en beneficio de determinados sectores campesinos.

\section{LA FRAGMENTACIÓN DE GRANDES PATRIMONIOS: EXPLORACIÓN DE HIPÓTESIS}

Para explicar el retroceso de los grandes patrimonios durante el período analizado, la historiografía ha barajado diversas hipótesis. Sin embargo, no todas se ajustan en la misma medida a lo que sucedió en el Baix Empordà. Por ello, dedicaremos este epígrafe a evaluar - aunque sea de forma breve y sintética- hasta qué punto, y de forma no excluyente, las explicaciones propuestas por distintos autores $\mathrm{y}$, mayoritariamente, para otras zonas pueden ayudarnos a comprender el retroceso de los grandes patrimonios bajoampurdaneses. Nos vamos a limitar a cuatro, que podemos definir como la hipótesis hereditaria, la hipótesis de la caída de la renta, la hipótesis de la pérdida relativa de rentabilidad y la hipótesis de los efectos de la conflictividad social.

Yvette Barbaza, en su estudio sobre la Costa Brava, consideró que la fragmentación de la gran propiedad debía atribuirse, en primera instancia, al debilitamiento del sistema hereditario tradicional, y en particular a la erosión del papel tradicional del bereu ${ }^{26}$. Según dicha autora, la obligación moral que todo bereu tenía de mantener y engrandecer la casa pairal fue convirtiéndose en una carga más que un honor, y la venta de masos -0

${ }^{25}$ Garrabou (1994).

26 Barbaza (1988), vol. II, p. 78. 
su reparto entre hermanos - se explicaría por el creciente desinterés de los herederos en ejercer su papel tradicional. Esta hipótesis también la recoge Ferrer, aunque con matices respecto a su formulación y a su prelación causal, quien relaciona las mutaciones en el sistema hereditario (y en particular el paso hacia un reparto igualitario) con la segregación de parcelas de los masos de la Cataluña central ${ }^{27}$. Debe advertirse, sin embargo, que el estudio de estos cambios en las pautas de transmisión hereditaria de las grandes propiedades durante la primera mitad del siglo XX ha recibido poca atención por parte de los especialistas y que los autores citados lo defienden como hipótesis más que resultado de una investigación específica.

Sin negar los efectos que sobre el mercado de la tierra pudieran haber causado los cambios en las estrategias hereditarias y, en particular, el abandono del modelo de familia troncal con sucesión unipersonal, existen indicios que su papel en el proceso que estamos analizando fue, en cualquier caso, secundario. Por varias razones. En primer lugar porque, con independencia del sistema hereditario vigente, el proceso de fragmentación de grandes propiedades y su transmisión hacia sectores campesinos fue general en Europa Occidental, donde dicho modelo era minoritario. En segundo lugar porque, si bien pueden detectarse algunas ventas relacionadas con la división de la herencia, la mayor parte están relacionadas con el pago de legítimas a hermanos menores. Al menos en el Baix Empordà existen pocas trazas de una modificación sustantiva del sistema hereditario entre el último tercio del siglo XIX y el primer tercio del siglo $\mathrm{Xx}$. Y porque, en tercer lugar, los problemas para pagar legítimas se produjeron en un contexto de dificultades económicas en los patrimonios rentistas. El pago de legítimas tradicionalmente había constituido una de las principales causas de endeudamiento de las familias de bisendats ${ }^{28}$. Que los problemas de liquidez para afrontar el pago de estos cargos favoreciesen la venta de tierras no era una novedad en términos históricos. El probable aumento de estas ventas sólo refleja la confluencia de los deberes del bereu con una mala situación económica. $Y$ es ésta la que debe ser analizada para hallar los motivos que indujeron a la fragmentación en los momentos críticos del ciclo patrimonial familiar.

La hipótesis de una caída sostenida de la renta adquiere, entonces, una dimensión relevante. Los estudios disponibles sobre la evolución de

\footnotetext{
${ }^{27}$ Ferrer (1991), p. 48.

${ }^{28}$ Bosch (1993), pp. 100-108, y Congost (1990), p. 226.
} 
esta variable durante la segunda mitad del siglo XIX y las primeras décadas del $\mathrm{xx}$ no son abundantes. El trabajo más importante de la historiografía española es el de Robledo sobre Castilla y León entre 1836 y 1913, a partir del cual se han realizado generalizaciones del impacto de la crisis finisecular sobre la renta para el conjunto español ${ }^{29}$. A diferencia de lo sucedido en países próximos, las series de arrendamientos castellanos muestran que la salida de la crisis se saldó con un refuerzo de la renta de la tierra, superando los niveles anteriores como consecuencia no sólo de las medidas arancelarias de sostenimiento de precios, sino también de la deforestación del monte y una mayor precarización y explotación de jornaleros y colonos. ¿Hasta qué punto estas conclusiones pueden extenderse a Cataluña y proyectarse hasta 1936? De ser aplicables, no sería correcto sostener que los grandes patrimonios experimentaron dificultades económicas. Sin embargo, los datos disponibles, tanto los correspondientes al Baix Empordà como los procedentes de otras comarcas catalanas, ni coinciden con la evolución castellana de las décadas de 1890 y 1900, ni confirman que su extrapolación para el conjunto del primer tercio del siglo $\mathrm{xx}$ sea razonable. Las series de renta del patrimonio Sentmenat correspondientes a las comarcas de Urgell y Vallès, aunque sólo alcanzan de forma fragmentaria hasta la primera década de nuestro siglo, muestran que la recuperación experimentada desde 1896 no significó el pleno restablecimiento de los niveles anteriores y, lo que es más importante, que éste se realizó a costa de una drástica reducción de los gastos de explotación, hecho de graves consecuencias futuras desde la perspectiva de la transformación y adecuación de las explotaciones a las nuevas condiciones ${ }^{30}$. Otra de las pocas series disponibles, correspondiente al patrimonio Maspons (Vallès Oriental), confirma que entre la segunda y la cuarta década del novecientos la evolución de la renta agraria tendió a la baja y que si la renta global se sostuvo fue gracias a los ingresos procedentes de la explotación forestal, los cuales, a pesar de su carácter espasmódico, significaron fuertes inyecciones compensatorias a una caída más o menos continuada de los ingresos agrarios ${ }^{31}$.

La serie de renta del patrimonio Bru (Gironès) entre 1885 y 1936 (aunque con un vacío central de veinte años), cuyas fincas eran muy próximas a los límites administrativos del Baix Empordà, también ratifica

\footnotetext{
29 Robledo (1984).

${ }^{30}$ Garrabou, Saguer y Sala (1993), pp. 115-116.

${ }^{31}$ Planas (1994), pp. 67 y $75-77$.
} 
la existencia de una caída de la renta a largo término ${ }^{32}$. A pesar que la familia Bru no fragmentó ni vendió su patrimonio, ejemplifica con bastante claridad lo que - según los datos disponibles- fue un fenómeno de amplitud más general. Su nivel de renta líquida se redujo en algo más de una tercera parte entre los años de la crisis agraria y la II República. Probablemente este descenso aún sería más acusado si contásemos con datos anteriores a la crítica década de 1880. Los esfuerzos realizados para atajar la caída tuvieron poco efecto. El aumento del gasto, buena parte del cual se dedicó a comprar ganado para el engorde, no fue suficiente. Y la intensificación de las talas en bosques y dehesas (deveses) sólo tuvo un leve efecto de amortiguación. En conjunto, la gestión del patrimonio se mantuvo bajo las fórmulas contractuales habituales (masoverías, aparcerías y arriendos monetarios) de forma bastante estable, aunque se apreció una cierta tendencia a aumentar la porción de tierras arrendadas en dinero, y ello a pesar del descenso de este capítulo en términos constantes. Una de las observaciones más relevantes que se obtienen de un análisis minucioso de los datos es la incapacidad que el patrimonio demostró para actualizar los arriendos monetarios al ritmo de la evolución general de los precios. Los arriendos monetarios se comportaron casi como una renta fija, prácticamente congelada, en un período de alza de precios. La desvalorización de los censos enfitéuticos, mayoritariamente monetarios, difícilmente podía haberse evitado. Sin embargo, ¿por qué se perdió capacidad para extraer el excedente campesino a través de los arriendos en dinero? ¿Y por qué se tendió a monetizar la renta de las pequeñas parcelas, en vez de mantenerlas bajo fórmulas parciarias? En resumen, los datos del patrimonio Bru constituyen un acertado ejemplo no sólo del proceso global de disminución de la renta, sino también de los diversos mecanismos que operaron en él y de las respuestas ambivalentes que los propietarios desarrollaron. Quizás las dos hipótesis que nos quedan por comentar nos permitan comprender algo más el comportamiento de estos propietarios.

${ }^{32}$ Los Bru fueron una familia no muy acaudalada de rentistas. Su domicilio -el mas Bru de Domeny - se encontraba a poca distancia de la ciudad de Girona. Su patrimonio - no muy extenso - estaba integrado por cinco masos dispersos en cinco localidades distintas, además de algunas piezas sueltas de bosque o dehesa. En total unas 110 hectáreas, cuyo ingreso bruto anual fue de aproximadamente 9.500 pesetas durante la década de 1880 . El grueso del patrimonio estaba cedido a masovería y sus producciones básicas - al menos en aquellas fincas que rentaban en especie - se reducían a poco más que los cultivos cerealicolas tradicionales, aunque puntualmente se aventuraron a ensayar la cria y engorde de ganado bajo fórmulas de aparceria pecuaria. 


\section{TABLA 1}

Evolución de la renta y composición del ingreso agro-forestal en el patrimonio Bru (1881-1936). Índice sobre la media anual en pesetas constantes de $1913\left(100=1881-90^{\mathrm{a}}\right)$

\begin{tabular}{rrrrrrrrrr}
\hline Periodo & $\begin{array}{c}\text { Arriendo } \\
\text { en especie }\end{array}$ & $\begin{array}{c}\text { Arriendo } \\
\text { monetario }\end{array}$ & Censos & Ganado & $\begin{array}{c}\text { Total } \\
\text { ingreso } \\
\text { agrario }\end{array}$ & Forestal & $\begin{array}{c}\text { Ingreso } \\
\text { agro- } \\
\text { forestal }\end{array}$ & $\begin{array}{c}\text { Gastos de } \\
\text { explota- } \\
\text { ción }\end{array}$ & $\begin{array}{c}\text { Renta } \\
\text { liquidac }\end{array}$ \\
\hline $1881-90^{\mathrm{a}}$ & 100 & 100 & 100 & 100 & 100 & 100 & 100 & 100 & 100 \\
$1891-00$ & 100 & 124 & 88 & 131 & 111 & 58 & 101 & 146 & 93 \\
$1901-10$ & 116 & 85 & 78 & 858 & 110 & 121 & 108 & 212 & 93 \\
$1911-20$ & - & - & - & - & - & - & - & - & - \\
$1921-30$ & 86 & 67 & 48 & 502 & 78 & 136 & 84 & 192 & 70 \\
$1931-36$ & 88 & 79 & 45 & 438 & 83 & 95 & 83 & 169 & 64 \\
\hline
\end{tabular}

${ }^{a}$ Los datos empiezan realmente en 1885 , aunque a efectos formales, para conservar una distribución en décadas, conste como año inicial 1881.

b No existen datos desde 1907 hasta 1922, inclusive.

" La renta líquida se ha obtenido deduciendo del ingreso los pagos en concepto de censos y contribuciones, además de los gastos de explotación. Una exposición más detallada del análisis contable y los conceptos utilizados en Garrabou, Saguer y Sala (1993).

FUENTE: Archivo particular de la familia Masó, libros de contabilidad.

Otra de las hipótesis que la historiografía ha esgrimido para razonar el retroceso de la propiedad rentista se centra en la pérdida de rentabilidad de las inversiones agrarias, en relación con otros tipos de inversión ${ }^{33}$. Ello explicaría la escasa presencia de grandes compradores en el mercado fundiario y la fragmentación de los masos en el momento de su venta. La baja atracción ejercida por el suelo agrario habría inhibido a los compradores rentistas y habría facilitado el acceso de los campesinos a la propiedad ${ }^{34}$. Este argumento tiene una doble virtud. Por una parte explica la ausencia de inversores burgueses y urbanos en el mercado de la tierra agrícola; pero también encaja en el comportamiento mostrado por los mismos hisendats, quienes tendieron a desplazar sus objetivos de inversión hacia otro tipo de activos. El patrimonio Bru vuelve a proporcionarnos una clara ilustración de ello. Tal como se observa en la tabla 2, los ingresos generados por ganancias obtenidas con acciones, depósitos bancarios, títulos de deuda, debitorios y censales aumentaron

\footnotetext{
${ }^{33}$ Barbaza (1988), vol. II, pp. 79, y Postel-Vinay (1974).

${ }^{34}$ Malatesta (1994), para una perspectiva europea.
} 
espectacularmente durante las primeras décadas del siglo XX. La familia Bru, que nunca había destacado por su actividad prestamista, consiguió reflotar e incluso mejorar su nivel de ingreso gracias al desplazamiento de sus inversiones hacia formas de renta mobiliaria, y en especial gracias a las ganancias especulativas obtenidas con títulos y acciones. Se estaba produciendo una transformación de la figura tradicional del rentista, hasta entonces ligada a la posesión de tierras.

\section{TABLA 2}

Composición del ingreso bruto ordinario del patrimonio Bru, 1881-1936 (en pesetas constantes de 1913)

\begin{tabular}{|c|c|c|c|c|c|}
\hline Periodo & $\begin{array}{c}\text { Ingreso } \\
\text { agrario } \\
\text { (porcentaje) }\end{array}$ & $\begin{array}{c}\text { Ingreso } \\
\text { forestal } \\
\text { (porcentaje) }\end{array}$ & $\begin{array}{l}\text { Ganancias } \\
\text { en activos } \\
\text { financieros } \\
\text { (porcentaje) }\end{array}$ & $\begin{array}{c}\text { Otros } \\
\text { ingresos } \\
\text { (porcentaje) }\end{array}$ & $\begin{array}{c}\text { Ingreso } \\
\text { anual } \\
\text { (pesetas) }\end{array}$ \\
\hline $1881-90 \ldots \ldots \ldots \ldots$ & 80 & 13 & 4 & 4 & 10.156 \\
\hline $1891-00 \ldots \ldots \ldots \ldots$ & 88 & 7 & 3 & 1 & 10.175 \\
\hline $1901-10 \ldots \ldots \ldots \ldots$ & 73 & 13 & 14 & 1 & 12.193 \\
\hline $1911-20 \ldots \ldots \ldots \ldots$ & - & 一 & 一 & - & - \\
\hline $1921-30 \ldots \ldots \ldots \ldots$ & 46 & 13 & 41 & 1 & 13.909 \\
\hline $1931-36 \ldots \ldots \ldots \ldots$ & 52 & 9 & 39 & 0 & 13.009 \\
\hline
\end{tabular}

Fuente: Archivo particular de la familia Masó, libros de contabilidad.

Esta transformación, sin embargo, no reposó únicamente sobre una superior rentabilidad de las inversiones realizadas fuera del sector agrario. No todo se reducía a ella. Históricamente, la ganancia no había sido el único móvil ni, quizás, el principal de la inversión rentista en tierras. La posesión de fincas había sido fuente de prestigio y de status; había constituido un signo de pertenencia a la élite. El Estado liberal del siglo XIX continuó manteniendo dicha tradición, estableciendo la propiedad como categoría básica de ciudadanía. Sólo a medida que la tierra dejó de ser la principal fuente de riqueza y la economía se diversificó, esta identificación entre élite y propiedad de la tierra se debilitó.

La inversión en tierra poseía, además, un segundo significado. En un contexto de gran volatilidad de la riqueza, antes del desarrollo del sistema bancario moderno, antes de que la deuda pública empezase a absorber 
porciones importantes del ahorro y sin temor a nuevas quiebras de la hacienda, la tierra era un valor seguro ${ }^{35}$. Daba lo mismo que el rendimiento obtenido fuese inferior si el capital invertido no se depreciaba ni se volatilizaba. La tierra era una forma de patrimonializar la riqueza, disminuyendo riesgos y asegurando su continuidad a lo largo del tiempo y de las generaciones. La atracción por la tierra no se basaba sólo, ni de forma prioritaria, en la rentabilidad monetaria. Por la misma razón, en la pérdida de interés hacia la tierra también jugaron una baza importante otros factores que la mera rentabilidad. En particular, el desarrollo del sistema financiero y bancario y la creciente seguridad de la deuda pública -que tendió a desplazar a la tierra como valor refugio- contribuyeron decisivamente al desvio de los potenciales grandes compradores de tierra hacia otros objetivos.

La última hipótesis que debemos examinar sostiene que la creciente conflictividad social del mundo rural pudo impulsar en alguna medida este proceso de transferencia de tierra hacia sectores campesinos. Los años del cambio de siglo fueron un período de consolidación de un nuevo movimiento campesino cualitativamente muy distinto de sus precedentes. El sufragio universal, la revisión de los contratos vitícolas después de la filoxera y la difusión de ideologías de transformación social dieron impulso a un movimiento con nuevos objetivos y nuevas formas de lucha ${ }^{36}$. ¿Hasta qué punto ello condicionó positivamente el proceso de transferencia de tierras? En Galicia, por ejemplo, se ha observado cómo la presión campesina contra la renta foral, articulada en un potente movimiento reivindicativo, fue decisiva en el proceso redencionista y en el acceso de los agricultores a la plena propiedad con anterioridad a la Ley de Redención de $1926^{37}$. Para algunas áreas vitícolas catalanas también se ha indicado que la creciente conflictividad social habría influido en las decisiones de venta de los propietarios de masos ${ }^{38}$. Pero a diferencia de la Cataluña central y meridional, el Baix Empordà se distinguió por un nivel de conflictividad agraria de tono menor. Hasta el advenimiento de la II República, la tensión social en el mundo rural ampurdanés fue comparativamente baja y estuvo hegemonizada por la versión más moderada y menos reivindicativa del sindicalismo agrario. ¿Cuestiona ello una relación estrecha entre conflictividad y venta de tierra a los campesinos? No necesariamente.

\footnotetext{
35 Kindleberger (1984), pp. 240-244.

36 Garrabou (1991).

37 Villares (1982), pp. 311 y ss.

38 Ferrer (1991) y Colomé (1996), pp. 250-260.
} 
Aunque la conflictividad local fuera menor, los propietarios bajoampurdaneses y gerundenses compartían el estado de alarma general. La literatura generada por los sindicatos católicos locales o el movimiento de constitución de asociaciones de propietarios con la finalidad explícita de defender la propiedad permiten pensar que los conflictos sobre tierras y rentas fueron vividos con menor distanciamiento del que podríamos suponer atendiendo sólo al nivel relativo de conflictividad. Tampoco debe olvidarse que bajo una apariencia de calma existían tensiones estructurales que se manifestaban con poca vistosidad, pero que cotidianamente recordaban que masoveros, arrendatarios, mozos y jornaleros no eran una fuerza de trabajo obediente y deferente ${ }^{39}$. Por otra parte, el sindicalismo católico hegemónico en la zona, a pesar de mantener un discurso basado en la bondad de la organización gerundense de la propiedad rural, también puede considerarse como la expresión de una nueva sensibilidad de determinados sectores propietarios partidarios de facilitar el acceso de los agricultores a la propiedad de la tierra. Aunque en términos efectivos la actividad de dichos sindicatos en el fomento de la propiedad campesina apenas superó el estadio testimonial, es razonable suponer que contribuyó en cierta medida a difundir esta sensibilidad sobre la bondad de la transferencia de tierras a cultivadores directos, siempre que se respetaran (y pagaran) los derechos de propiedad. Ello no implica que sus efectos sobre el mercado de la tierra fueran relevantes. No es fácil demostrar que la conflictividad y la presión social impulsasen de forma significativa la venta fraccionada de grandes propiedades a campesinos. Incluso sería relativamente simple -demasiado simple - argumentar lo contrario: en el momento de máxima intensificación de la conflictividad agraria, durante la II República, el proceso de transferencia se redujo considerablemente. Por el contrario, es más razonable pensar que la conflictividad pudo contribuir a inhibir - un motivo más- la presencia de grandes compradores rentistas. La experiencia común muestra que la inversión se aleja de aquellos sectores con un nivel elevado de conflictividad.

En resumen, si jerarquizásemos los factores que tuvieron alguna incidencia sobre el comportamiento de los grandes propietarios en el mercado de la tierra, los datos disponibles apuntarían hacia la preeminencia de la variable renta de la tierra, aunque la base empírica que sustenta las conclusiones sobre dicha variable deba ser ampliada. La erosión sufrida por la renta de la tierra, la pérdida relativa de rentabilidad y la depreciación

\footnotetext{
${ }^{39}$ Howkins (1977).
} 
de algunos valores que hasta entonces habían hecho atractiva la inversión en tierras (status, seguridad) configuran un contexto que no sólo estimuló la fragmentación y venta de masos, sino también la ausencia de grandes inversores que sustituyeran a los hisendats, cuya presencia retrocedía. A ello también contribuyó, aunque a mayor distancia, la alarma de los propietarios ante un creciente clima de confrontación social. Por el contrario, más oscuro es el papel que pudieron desempeñar las aún no demostradas modificaciones en las pautas de transmisión hereditaria en el seno de las familias propietarias.

\section{CAMPESINOS EN EL MERCADO DE LA TIERRA}

¿Qué condiciones hicieron posible el avance de un grupo significativo de patrimonios campesinos en dicho contexto? ¿Cómo explicar que, en un marco general de caída relativa de los precios agrícolas y de deterioro de los términos de intercambio del sector agrario, muchas familias campesinas adquirieran extensiones de tierra importantes en relación con el tamaño inicial de su patrimonio y con sus posibilidades de ingreso? Existen algunos elementos a primera vista chocantes que deben incitarnos a pensar sobre las singularidades de la posición y las estrategias de las unidades campesinas. Aunque no expliquen directamente el cómo y el porqué de las compras campesinas, pueden orientarnos hacia una explicación más global y comprensiva.

La cronología de las sucesivas olas de emigración y despoblación rural es uno de ellos. ¿No es paradójico que la fase más intensa de éxodo rural bajoampurdanés corresponda al período intercensal 1857-1877 ( $-0,6$ por 100 anual), coincidiendo con un ciclo agrario no depresivo? La población rural comarcal volvió a decrecer durante los años de la crisis agraria (período intercensal 1887-1900) y entre 1920 y 1936, pero a un ritmo considerablemente inferior $(-0,38$ por 100 y $-0,33$ por 100 , respectivamente). Además, la pérdida de población de 1887-1900 fue completamente restituida por un movimiento de signo contrario durante la primera década del nuevo siglo ( $+0,5$ por 100 anual). De la evolución demográfica puede deducirse que la dependencia de la población rural bajoampurdanesa respecto de la coyuntura agraria no era tan estrecha como cabría suponer. Por contra, pensamos que pone de relieve el vínculo que las unidades familiares campesinas mantenían con una amplia gama de actividades artesanales y forestales; actividades que durante la segunda 
mitad del siglo XIX sufrieron profundamente los efectos $-\mathrm{y}$ también los estímulos- de la expansión fabril, de la mecanización del trabajo y de las mejoras en la red de transporte y comunicaciones. La crisis artesanal que afectó a villas y ciudades también repercutió sobre las economías campesinas, caracterizadas en buena medida por aquello que la historiografia francesa ha bautizado como pluriactividad. Es significativo en este sentido que las localidades bajoampurdanesas con mayor abundancia de recursos (forestales, minerales...) y, por ende, mayores posibilidades de diversificación ocupacional fueran también las que más población perdieran entre 1857 y 1877.

\section{GRÁFICO 2}

Cronología de los préstamos hipotecarios contraídos por campesinos, menestrales, jornaleros y artesanos sobre tierras de Ullastret (1870-1909)

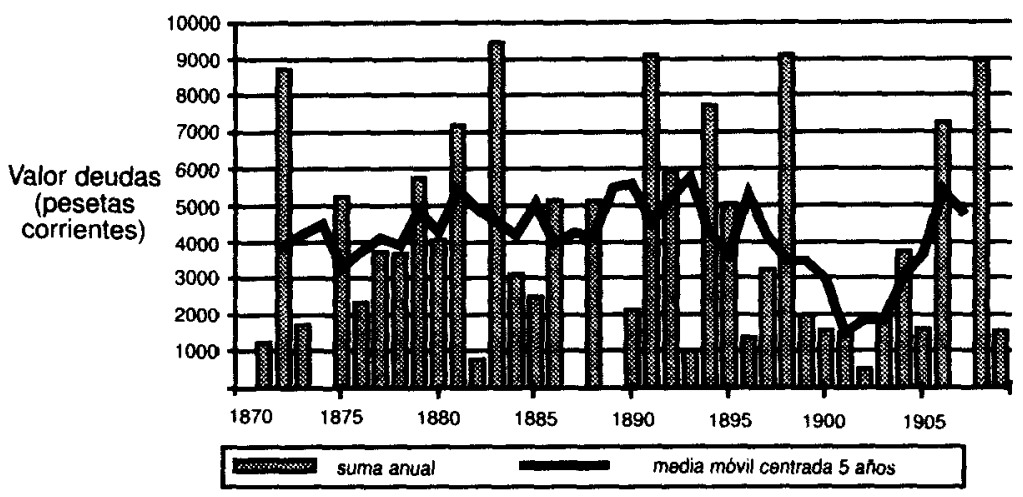

FuEnTE: Registro de la Propiedad, Ullastret.

Otro elemento que debe motivarnos a reflexionar sobre el carácter de las familias campesinas en tanto que unidades económicas es su trayectoria durante los años de la crisis agraria finisecular. Es cierto que buena parte de la relevancia de esta crisis no se debió tanto a sus efectos inmediatos como a los efectos en el medio y largo plazo y a su carácter de ruptura respecto a las condiciones anteriores ${ }^{40}$. Sin embargo, en el

\footnotetext{
${ }^{40}$ Garrabou (1994).
} 
Baix Empordà, es hasta cierto punto sorprendente el tono atenuado de sus consecuencias inmediatas en términos de endeudamiento campesino, de desposesión e incluso de expulsión demográfica. Sin pretensión de minimizarlas, uno podría esperar que las consecuencias sociales fueran de mayor entidad, atendiendo al papel otorgado a la crisis. Pero, por ejemplo, una variable tan sensible a los efectos de la coyuntura como el endeudamiento campesino no parece registrar alteraciones de magnitud. El valor y número de préstamos hipotecarios contraídos por campesinos, artesanos y jornaleros, si bien experimentó una cierta elevación durante la crisis, ésta fue de escasa entidad. Y las ventas a carta de gracia, el último eslabón de la cadena crediticia, a pesar de mostrar también un cierto incremento movilizaron extensiones prácticamente insignificantes. Quizás allí donde más se perciban los efectos de la crisis sea en la cancelación de deudas más que en la creación de nuevos préstamos. Si hasta la década de 1890 las cantidades prestadas y las devueltas tendieron a equipararse, entre 1890 y 1900 este equilibrio se rompió y la devolución de muchos préstamos se prolongó hasta la década siguiente. La impresión general, sin embargo, es que el nivel de endeudamiento campesino, si bien fue sensible a un empeoramiento de las condiciones, no implicó ni tradujo una desestabilización traumática de las precarias economías familiares campesinas. Exploraciones realizadas en Galicia por L. Domínguez parecen apuntar en el mismo sentido ${ }^{41}$. Igualmente, durante aquellos años se produjo un cierto incremento en la venta de tierras por parte de campesinos, menestrales, artesanos y jornaleros; pero tampoco fue un aumento espectacular, en especial si tomamos como referencia los periodos posteriores. Además, los campesinos no sólo aumentaron sus ventas, sino también sus compras. En cierta medida la desposesión de algunas familias campesinas se compensó sobradamente con las adquisiciones de otras.

¿Cuáles fueron los mecanismos que, además de dotar de inmunidad a muchas unidades familiares campesinas ante la crisis finisecular, también hicieron posible un cierto proceso de acumulación territorial entre 1880 y 1940? No estamos aún en condiciones de aportar respuestas definitivas, ni tampoco de identificar con precisión cuáles fueron los sectores campesinos que compraron tierras, pero creemos que debemos partir de una comprensión de la posición, las estrategias y los objetivos económicos de los distintos tipos de unidades campesinas existentes. En este sentido, se pueden barajar tres elementos que, de forma combinada, desempeñaron

${ }^{41}$ Citado por Villares y Fernández Prieto (1992), pp. 91-92. 
un papel relevante en este proceso: un cierto mantenimiento de estrategias de producción para el autoconsumo, la existencia de un flujo de ingresos salariales y/o extraagrícolas en los presupuestos domésticos campesinos y, finalmente, una amplia participación de las pequeñas unidades campesinas en la modernización y reorientación productiva del sector.

No hay duda que, en términos genéricos, las estrategias de producción para el autoconsumo implicaban una protección de las unidades campesinas ante las contingencias de los precios. Podríamos pensar, en consecuencia, que el mantenimiento de dichas estrategias podría explicar —en algunos momentos, y particularmente en el período de la crisis finisecular- su resistencia ante el deterioro de la situación económica. Sin embargo, éste es un tema complejo, sobre el cual aún disponemos de una base empírica débil. Y ello a pesar que el debate sobre el comportamiento de los campesinos como agentes económicos se haya centrado, en buena parte, en la contraposición entre una economía campesina tradicional cuyo objetivo era la autosatisfacción de las necesidades familiares y una agricultura capitalista plenamente mercantilizada. Es difícil negar que, a fines del siglo XIX, la vinculación de las unidades familiares campesinas al mercado hubiera avanzado significativamente. Aunque debe tenerse en cuenta que estos progresos probablemente no habían sido uniformes ni homogéneos. La posición de un pequeño campesino o del masover de un mas importante ante el mercado no eran la misma. No todos los que cultivaban la tierra compartían los mismos objetivos económicos ni participaban en la misma medida en los intercambios mercantiles. Los escasos testimonios disponibles -obtenidos mediante una pequeña encuesta oral ${ }^{42}$ - nos permiten sospechar que, en términos generales, a medida que el tamaño de la explotación se reducía la proporción de producto agrícola autoconsumido por la unidad familiar solía incrementarse (con excepciones como el caso de las explotaciones vitícolas), y viceversa. Ello no tiene nada que ver con su autosuficiencia, sino con las proporciones de producto vertidas al mercado. Las familias con menor grado de autosuficiencia probablemente eran las que menores cantidades (relativas o absolutas) de producto agrícola comercializaban y mayor proporción autoconsumían. En la medida en que la existencia de esta gradación sea cierta, cabría pensar en una menor vulnerabilidad de muchos pequeños campesinos a las coyunturas de los precios agrarios. Ello -la escasa sensibilidad a las fluctuaciones de los precios agrícolas- es importante

${ }^{42}$ Reproducidas en Saguer (1996), pp. 657-689. 
para comprender la trayectoria de muchas pequeñas unidades campesinas durante la crisis finisecular. Con posterioridad, paralelamente al desarrollo de nuevas orientaciones productivas del sector, esta situación fue modificándose lentamente.

Es incuestionable, de todos modos, que los vínculos con el mercado existían en todos los estratos de la sociedad rural. Entre otras razones porque para la inmensa mayoría de unidades familiares campesinas la autosuficiencia a partir de producciones propias era un objetivo inalcanzable. La pequeñez de sus explotaciones no lo permitía. Los vínculos con el mercado eran importantes también como consumidores, pero sobre todo como trabajadores. Uno de los elementos básicos que permite entender tanto el tipo de dependencia establecido con el mercado como la resistencia ante la crisis agraria finisecular lo constituye la posibilidad de remuneración fuera del sector agrario: bien fuera como asalariado; bien como productor independiente integrado en una red de comercialización. Probablemente no fue tanto la desvinculación como el tipo de vínculo con el mercado lo que dio a muchas unidades campesinas una mayor capacidad de resistencia. Muchos indicios apuntan hacia un modelo de pequeña explotación familiar relativamente cerrada en ella misma, con un fuerte excedente laboral orientado bien hacia la realización de actividades no agrarias organizadas en el seno de la misma unidad familiar, bien hacia la venta de trabajo sobrante a cambio de un salario. Estos ingresos extraagrícolas y salariales significaban buena parte del ingreso monetario de las familias rurales. De ahí procedían buena parte de los recursos empleados en la compra de tierras.

Ello tiene una relevancia especial dado que la evolución del salario real rural desde la década de 1870 experimentó una notable tendencia al alza, alejándose de forma creciente de la evolución seguida por los precios agrícolas ${ }^{43}$. Dicha tendencia se mantuvo, con algunas modulaciones, hasta la Primera Guerra Mundial, momento en el cual las series salariales disponibles muestran un brusco aumento no sólo en términos nominales, sino también, y sobre todo, reales. Aunque cabría analizar con mayor detenimiento las condiciones de ocupación, en términos generales puede afirmarse que la evolución salarial habría jugado a favor de este amplio segmento de pequeñas unidades campesinas. En cambio, habría perjudicado notablemente las explotaciones de mayores dimensiones que requerían la contratación de trabajo asalariado complementario al familiar.

${ }^{43}$ Garrabou, Pujol y Colomé (1991). 
En ellas las posibilidades de ingreso neto habrían mermado por el doble efecto de la disminución - absoluta o relativa - de los precios agrícolas y la rigidez del salario. Para las pequeñas unidades campesinas, a medio término, los ingresos salariales pudieron tener un papel clave en las posibilidades no sólo de reproducción económica, sino también de acumulación y ahorro. El acceso a la propiedad habría sido propiciado por la disponibilidad de unos ahorros acumulados mediante trabajos realizados fuera $o$ al margen de la explotación ${ }^{44}$. Aunque no parecen haber tenido importancia en el Baix Empordà, las remesas de familiares emigrados - cuando las había - podían haber reforzado esta acumulación de ahorros $^{45}$. En cualquier caso, difícilmente puede comprenderse el acceso de pequeños campesinos a la compra de tierra si no se tiene en cuenta la evolución al alza del salario rural.

Finalmente, también cabe considerar el papel desempeñado por la participación de las pequeñas unidades campesinas en la transformación y modernización de las estructuras productivas del sector agrario bajoampurdanés. Para muchas unidades familiares, la incorporación de determinados cambios incrementó la posibilidad de aumentar los excedentes para el mercado. Es plausible que ciertas coyunturas favorables, como la acaecida durante la Primera Guerra y posguerra Mundial, pudieran aumentar sus posibilidades de acumulación patrimonial.

La transformación de la agricultura bajoampurdanesa con posterioridad a los años de la crisis finisecular se orientó claramente hacia la producción pecuaria. El olivo $\mathrm{y}$, especialmente, la viña retrocedieron considerablemente, a la par que se expandían aquellos cultivos y rotaciones que tenían como efecto multiplicar forrajes, piensos y otros alimentos para el ganado. Los cultivos de regadío experimentaron un crecimiento bastante espectacular, especialmente en los municipios del Bajo Ter, donde la recuperación del arroz favoreció no sólo a los grandes propietarios, sino también a pequeños y medianos campesinos agrupados en comunidades de regantes. Sin embargo, insistimos, el eje principal de transformación del sector fue la expansión de los cultivos para alimentación animal y, en estrecha relación con ello, la expansión de la ganadería. La evolución de los precios relativos favoreció dicha reorientación. Se produjo una ruptura respecto al modelo cerealícola ochocentista, que giraba en torno a los cereales panificables,

\footnotetext{
${ }^{44}$ Hubscher (1985), pp. 14 y ss., y Massullo (1990).

45 Villares (1982), pp. 396-415.
} 


\section{GRÁFICO 3}

Índices del precio de la carne y el trigo (1840-1935)

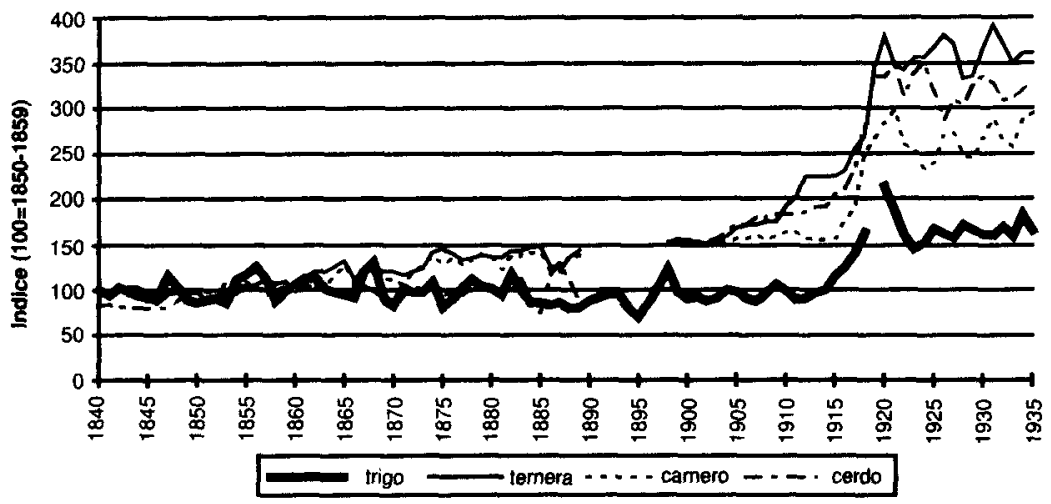

FUENTE: Índice elaborado a partir de la serie de precios del trigo del mercado de Figueras (1840-1935) publicada por Armengol (1980), las series de ternera, carnero y cerdo fresco del mercado de Girona (1840-1889), y las series de ternera, carnero y cerdo fresco de Barcelona reproducidos por Pujol (1988).

reduciéndose notablemente la superficie dedicada a trigo y tranquillón (mestall) en beneficio de forrajes, piensos, raíces y tubérculos. La expansión de la ganadería fue acompañada por una recomposición del peso de las distintas especies. En términos generales, se produjo un desplazamiento del ganado de trabajo por ganado de renta; destacando especialmente, dentro del segundo grupo, el progreso experimentado por el ganado vacuno y el porcino. No es en vano que cuando, en 1938, dos profesores de la Escuela Superior de Agricultura de Cataluña (J. Llovet y J. M. Riu) escogieron un modelo de explotación representativa de la comarca para su estudio sobre las características económicas de la agricultura catalana éste fuera el de una explotación regable con agua del Ter, cultivada a base de cereales y forrajes consumidos íntegramente por el ganado bovino, ovino y porcino de la finca, y que, además, comprendía una pequeña superficie de olivar en la montaña. En este modelo se estaban sintetizando las principales líneas de cambio del primer tercio de siglo.

En cualquier caso, lo que nos interesa destacar es que esta transformación no fue exclusiva de los campesinos más acomodados ni 
de los arrendatarios de masos. Es más, mientras muchos masovers tuvieron dificultades para desarrollar a fondo las nuevas orientaciones productivas, muchos pequeños propietarios, con la voluntad de intensificar al máximo el producto de sus fincas, participaron activamente en el proceso de cambio agrario $^{46}$. Es significativo, en este sentido, que las dos especies ganaderas en expansión (vacuno y porcino) fueran las que tradicionalmente habían estado vinculadas a la pequeña explotación campesina (a diferencia, por ejemplo, del ovino). Por otra parte, numerosos testimonios apuntan a que si la vinculación al mercado de las unidades familiares campesinas tuvo alguna expresión realmente importante ésta fue la venta de animales. Buena parte de los ingresos obtenidos por las pequeñas explotaciones con la venta de su producción procedían de la comercialización de animales, hecho que tendió a incrementarse con posterioridad a la crisis finisecular. La favorable evolución del precio de la carne en relación con el precio de los cereales y el hecho que la producción pecuaria fuese la producción comercial por excelencia en las unidades familiares campesinas permite explicar con mayor claridad sus posibilidades de acumulación. Precisamente las dos especies que mayor difusión experimentaron y que con mayor facilidad se adecuaron a la estructura de la pequeña y mediana explotación campesina fueron las que experimentaron un alza más acentuada en sus cotizaciones a largo plazo.

\section{CONCLUSIÓN}

El avance de la propiedad campesina posterior a la crisis finisecular ha sido valorado de formas distintas. Como en otros debates historiográficos también aquí podemos encontrar pesimistas y optimistas. Massullo (1990), por ejemplo, plantea el avance de la piccola propietà contadina en términos altamente positivos y califica de epopeya la fuerte expansión de ésta durante las primeras décadas del siglo xx. Gervais, Jollivet y Tavernier (1977) lo califican de triunfo de la explotación familiar, remarcando los aspectos positivos tanto en términos económicos como sociales. Grigg (1989), por el contrario, adopta una posición más matizada y advierte que «the consequences of land purchase were not always bappy for the new owners». Aunque nuestro argumento haya destacado el avance de la propiedad campesina como línea de fuerza central, no es nuestra intención apostar

46 Sobre ello véase Villares y Fernández Prieto (1992). 
por una interpretación demasiado triunfalista ni optimista. Por ello, no podemos concluir sin remarcar que el proceso tuvo un carácter ambivalente y que el avance de unos fue acompañado por la expulsión de otros. Tampoco debemos olvidar que dicho avance de la propiedad campesina no fue un proceso simple ni indoloro. La capacidad de adecuación mostrada por las pequeñas explotaciones campesinas se basaba, en primer lugar, en su predisposición para aceptar niveles de remuneración inferiores a los estándares. A ello se referían los contemporáneos cuando contraponían las cuentas de payés a las cuentas de comercio. Aunque la autoexplotación campesina -por expresarlo en términos convencionales- no fue el único mecanismo de reajuste de las unidades campesinas, no debemos olvidar que también tuvo su papel.

\section{BIBLIOGRAFÍA}

ARMENGOL, D. (1980): «La agricultura en el Alto Ampurdán a mediados del siglo XIX», Annals de l'Institut d'Estudis Empordanesos, núm. 14, pp. 9-137.

BarbazA, Y. (1988): El paisatge bumà de la Costa Brava, Barcelona, Edicions 62. Bosch, M. (1993): Casa Carles. Evolució socio-econòmica d'una familia d'bisendats gironins a la primera meitat del segle XIX, Universitat Pompeu Fabra, Institut Universitari d'Història Jaume Vicens Vives, trabajo de investigación de tercer ciclo.

Calatayud, S. (1989): Capitalismo agrario y propiedad campesina. La Ribera del Xúquer, 1860/1930, Valencia, Edicions Alfons el Magnànim/Institució Valenciana d'Estudis i Investigació.

Cela, C. J. (1979): Capitalismo y campesinado en la isla de Mallorca, Madrid, Siglo XXI.

Colomé, J. (1992): «Estructura de la propiedad y crisis agraria a finales del siglo XIX: la comarca del Penedès», en GarrabOu (1992), pp. 237-260.

- (1996): L'especialització vitícola a la Catalunya del segle XIX. La comarca del Penedès, Universidad de Barcelona, tesis doctoral.

ConGost, R. (1981): «Notes sobre la propietat de la terra a les comarques gironines durant la segona meitat del segle xIX», Estudi General, núm. 1, pp. 211-221.

- (1990): Els propietaris $i$ els altres. La regió de Girona, 1768-1862, Vic, Eumo.

FERRER, Ll. (1987): Pagesos, rabassaires $i$ industrials a la Catalunya Central (segles XVIII.XIX), Publicacions de l'Abadia de Montserrat.

- (1991): «La pequeña explotación en un viñedo de la Cataluña central en los siglos XIX y xx. Consideraciones sobre su evolución y comportamiento», en SaAVEDRA y Villares (1991), vol. 2, pp. 34-62.

Garrabou, R. (1991): «La conflictivitat pagesa a Catalunya i al País Valencià a l'època contemporània», Miscel.lània d'bomenatge a Josep Benet, Publicacions de l'Abadia de Montserrat, pp. 81-111. 
- (coord.) (1992): Propiedad y explotación campesina en la España contemporánea, Madrid, MAPA/Institut d'Estudis Menorquins.

- Saguer, E., y SalA, P. (1993): «Formas de gestión patrimonial y evolución de la renta a partir del análisis de contabilidades agraria: los patrimonios del marqués de Sentmenat en el Vallés y en Urgell (1820-1917)», Noticiario de Historia Agraria, núm. 5, pp. 97-123.

- (1994): «Trasformazioni strutturali dell'agricoltura europea durante la crisi: analisi del caso spagnolom, AA. VV., L'agricoltura in Europa e la nascita della questione agraria, Roma, Istituto Alcide Cervi/Dedalo, pp. 31-52.

- Pujol, J., y Colomé, J. (1991): «Salaris, ús i explotació de la força de treball agrícola (Catalunya, 1818-1936)», Recerques, núm. 24, pp. 23-51.

Gervais, M.; Jollivet, M., y Tavernier, Y. (1977): «La fin de la France paysanne», en Duby ET Wallon (dir.) Histoire de la France rurale, núm. 4, Paris, Seuil.

González de Molina, M., y Sevilla Guzmán, E. (1991): «Minifundio y gran propiedad agraria: estabilidad y cambio en la Alta Andalucía, 1758-1930», en SaAvedra y Villares (1991), vol. 2, pp. 88-138.

Goodman, D., y ReDClifT, M. (1991): Refashioning Nature. Food, Ecology and Culture, London, Routledge.

GrIGG, D. (1989): English Agriculture. An Historical Perspective, Oxford, Blackwell.

Grupo de Historia Agraria de Murcia (1992): «La propiedad de la tierra en los regadíos del Segura durante los siglos XIX y XX», en GaRrabou (1992), pp. 159-190.

HowkINS, A. (1977): «Structural Conflict and the Farmworker: Norfolk, 1900-1920», Joumal of Peasant Studies, 4, 3, pp. 217-229.

HubSCHER, R. (1985): «La petite exploitation en France: Reproduction et competitivité (fin $\mathrm{XXX}^{e}$ siècle-début $\mathrm{XX}^{e}$ siècle)», Annales $E S C$, año 40, núm. 1, pp. 3-34.

KaraOUZOU, E. (1993): «Las reformas agrarias en Grecia, siglos XIX y XX», Noticiario de Historia Agraria, núm. 6, pp. 59-83.

KAUTSKY, K. (1899): La cuestión agraria. Estudio de las tendencias de la agricultura moderna y de la política agraria de la socialdemocracia, Barcelona, Laia, 1974.

KINDLEBERGER, C. P. (1984): Historia financiera de Europa, Barcelona, Crítica, 1988.

Koning, N. (1996): The Failure of Agrarian Capitalism. Agrarian Politics in the United Kingdom, Germany, the Netherlands and the USA, 1846-1919, London, Routledge.

LLOBET, S. (1955): «De geografía agraria en la comarca del Maresme (Barcelona)», Estudios geográficos, núm. 58, pp. 23-71, y núm. 59, pp. 215-297.

MALATESTA, M. (1994): «La deruralizzacione delle élites. Il caso italiano nel contesto europeo, 1800-1914», Annali 1993/1994, Istituto Alcide Cervi, pp. 139-176.

Massullo, G. (1990): «Contadini. La piccola propietà coltivatrice nell'Italia contemporanea», en BEVILACQUA (a cura di), Storia dell'agricoltura italiana in età contemporanea, Venezia, Marsilio.

MnláN, J., y Calatayud, S. (1992): «Formas de explotación y mercados de trabajo en la agricultura valenciana de regadí (1800-1930)», en GARRABOU (1992), pp. 325-349.

OfFer, A. (1989): The First World War. An Agrarian Interpretation, Oxford, Clarendon. 
Planas Maresma, J. (1994): «Agrarisme i gestió de la propietat al primer terç del segle xx. Un exemple significatiu: Jaume Maspons i Camarasa (1872-1934)», Estudis d'Historia Agrària, núm. 10, pp. 57-83.

Postel-VInAY, G. (1974): La rente foncièrie dans le capitalisme agricole. Analyse de la voie classique du dévelopment du capitalisme dans l'agriculture à partir de l'exemple du Saissonnais, Paris, Maspero.

PUjoL, J. (1988): Les transformacions del sector agrari català entre la crisi finisecular y la guerra civil, Universitat Autònoma de Barcelona, tesis doctoral.

ROBLEDO, R. (1981): «Movilidad de la propiedad de la tierra en una zona castellana (1861-1960)», Estudi General, núm. 1, pp. 223-230.

- (1983): «Notas sobre el precio de la tierra en España (1836-1914)», Revista de Historia Económica, I, 2, pp. 253-275.

- (1984): La renta de la tierra en Castilla y León, 1836-1913, Madrid, Banco de España.

- (1985): «Los arrendamientos castellanos antes y después de la crisis de fines del siglo XIX», en Garrabou y SAnz (eds.), Historia Agraria de la España Contemporánea, vol. 2, Expansión y crisis (1850-1900), Barcelona, Crítica, pp. 369-411.

Román Cervantes, C. (1994): «Evolución de los precios de la tierra en un mercado local: la comarca del Campo de Cartagena (1866-1974)», Revista de Historia Económica, XII, 1, pp. 145-171.

SaAvedra, P., y Villares, R. (eds.) (1991): Señores y campesinos en la Peninsula Ibérica, siglos XVIII XX, Barcelona, Crítica.

SABIO, A. (1992): «Las compraventas de tierra, una faceta de la integración mercantil forzosa del pequeño campesinado. Cariñena, 1886-1934», Noticiario de Historia Agraria, núm. 4, pp. 315-334.

- (1996): Los mercados informales de crédito y tierra en una comunidad rural aragonesa (1850-1930), Madrid, Banco de España.

SAGuer, E. (1996): La consolidació de la propietat pagesa a Catalunya (El Baix Empordà, 1850-1940), Girona, Universitat de Girona, tesis doctoral en microficha.

- (1997): «La consolidación de la propiedad campesina en Cataluña. Un análisis cuantitativo de las transformaciones en la propiedad de la tierra (El Baix Empordà, 1860-194)», en prensa.

SAlES, F. (1994): «Transformacions agràries a les Garrigues (1860-1936)», Recerques, núm. 29, pp. 87-108.

SegurA, A. (1993): Burgesia $i$ propietat de la terra a Catalunya en el segle XIX. Les comarques barcelonines, Barcelona, Curial.

Thompson, F. M. L. (1966): «The Social Distribution of Landed Property in England since the Sixteenth Century», EHR, XIX, 3, pp. 505-517.

TRIBÓ, G. (1990): Evolució de l'estructura agrària del Baix Llobregat (1860-1931), Universitat de Barcelona, tesis doctoral.

- (1992): «Evolución de la estructura agraria del Baix Llobregat (1860-1930)», en Garrabou (1992), pp. 209-236. 
Villares, R. (1982): La propiedad de la tierra en Galicia, 1500-1936, Madrid, Siglo XXI.

- y Fernández Prieto, L. (1992): «La crisi agrària del final del segle XIX i l'adaptació de l'explotació pagesa gallega», Recerques, núm. 26, pp. 89-106. 